\title{
Discectomia endoscópica transforaminal lombar: Resultados clínicos e complicações*
}

\section{Transforaminal Endoscopic Lumbar Discectomy: Clinical Outcomes and Complications}

Leonardo Yukio Jorge Asano ${ }^{1}$ João Paulo Machado Bergamaschi ${ }^{2}$ Álvaro Dowling ${ }^{2}$ Luciano Miller Reis Rodrigues ${ }^{1}$

${ }^{1}$ Faculdade de Medicina do ABC, Santo André, SP, Brasil

${ }^{2}$ Clínica Kennedy, São Paulo, SP, Brasil

Rev Bras Ortop 2020;55(1):48-53.
Endereço para correspondência Leonardo Yukio Jorge Asano, Faculdade de Medicina do ABC, Avenida Príncipe de Gales 821, Vila Príncipe de Gales, Santo André, SP, 09060-650, Brasil (e-mail: Yukioja@hotmail.com).

\section{Resumo \\ Palavras-chave \\ - hérnia de disco \\ - discectomia percutânea \\ - endoscopia \\ - procedimentos cirúrgicos minimamente invasivos}

\section{Abstract}

Objetivo Avaliar os resultados clínicos e funcionais da discectomia endoscópica transforaminal lombar.

Materiais e Métodos De agosto de 2015 a janeiro de 2017, 101 pacientes portadores de hérnia de disco lombar refratária ao tratamento clínico foram submetidos a discectomia endoscópica. Por meio de avaliação clínica pela Escala Visual Analógica e análise funcional pelo questionário Oswestry Disability Index, os pacientes foram analisados no período pré-operatório, no pós-operatório imediato, com 1 mês, 3 meses, 6 meses e 1 ano após a cirurgia.

Resultados A média de idade dos participantes foi de 48.1 anos. Os níveis discais mais acometidos foram L4-L5, seguidos de L5-S1. Um total de 29 pacientes foram abordados em 2 níveis discais. Após 1 mês de seguimento pós-operatório, a média das pontuações nos questionários (EVA e ODI) diminuiu significativamente $(p<0.001)$.

Conclusão A discectomia endoscópica transforaminal lombar mostrou ser uma alternativa segura, eficaz e minimamente invasiva para o tratamento de hérnia de disco lombar. O procedimento tem vantagens, como curto período de internação hospitalar, cirurgia realizada sob anestesia local e sedação, retorno precoce às atividades diárias, e baixa taxa de complicações.

Objective To evaluate the clinical and functional results of transforaminal endoscopic lumbar discectomy.

Materials and Methods From August 2015 to January 2017, 101 patients with lumbar disc hernia refractory to clinical treatment underwent endoscopic discectomy. Through clinical evaluation by the Visual Analogue Scale and functional evaluation by the Oswestry Disability Index questionnaire, the patients were analyzed in the

\footnotetext{
Trabalho Desenvolvido na Faculdade de Medicina do ABC, Santo André, SP, Brasil.
}

recebido

20 de Setembro de 2018 aceito 22 de Janeiro de 2019
DOI https://doi.org/

$10.1055 / \mathrm{s}-0039-1700822$. ISSN $0102-3616$
Copyright $\odot 2020$ by Sociedade Brasileira License terms de Ortopedia e Traumatologia. Published by Thieme Revinter Publicações Ltda, Rio de Janeiro, Brazil 


\section{Keywords}

- intervertebral disc displacement

- percutaneous discectomy

- endoscopy

- minimally-invasive surgical procedures preoperative period, in the immediate postoperative period, at 1 month, 3 months, 6 months and 1 year after surgery.

Results The mean age of the participants was 48.1 years. The most affected disc levels were L4-L5 and L5-S1. A total of 29 patients were treated at 2 disc levels. After 1 month of postoperative follow-up, the mean scores on the questionnaires (EVA and ODI) decreased significantly $(p<0.001)$.

Conclusion Transforaminal endoscopic lumbar discectomy has been shown to be a safe, effective and minimally-invasive alternative for the treatment of lumbar disc herniation. The procedure has advantages, such as short hospital stay, surgery performed under local anesthesia and sedation, early return to daily activities, and low rate of complications.

\section{Introdução}

Cerca de $60 \%$ a $80 \%$ dos adultos podem experimentar dor lombar em algum momento da vida. ${ }^{1}$ Com a idade de 30 anos, quase metade dos adultos experimentam um episódio substantivo de dor lombar. ${ }^{2}$ Hérnia de disco lombar é uma causa comum de dor lombar e ciatalgia, com uma incidência anual de 5 casos por mil pessoas. ${ }^{3}$ Atualmente, a hérnia discal lombar é a condição que mais leva à cirurgia de coluna, principalmente em homens por volta dos 40 anos de idade. ${ }^{4}$

Em 1934, Mixter e Barr ${ }^{5}$ foram os primeiros a descrever a técnica de discectomia convencional aberta, que poderia aliviar a dor e melhorar a função do nervo. Em 1989, Hijikata ${ }^{6}$ realizou uma nucleotomia por meio de uma cânula inserida no centro do disco intervertebral por um acesso posterolateral. Ele relatou um achado satisfatório pós-operatório em $64 \%$ dos pacientes. Kambin e Schaffer, ${ }^{7}$ em 1989 , utilizaram um artroscópio para visualização e excisão do disco. Foley e Smith, ${ }^{8}$ em 1997, e Jhala e Mistry, ${ }^{9}$ em 2010, introduziram a técnica de discectomia endoscópica, e relataram achados clínicos satisfatórios em 100 pacientes. Yeung ${ }^{10}$ desenvolveu um canal de trabalho endoscópico rígido para discectomia endoscópica lombar percutânea.

A discectomia endoscópica lombar é uma técnica minimamente invasiva para hérnias de disco que tem diversas vantagens, como menor dano tecidual, com preservação da musculatura paravertebral, menor tempo de hospitalização, menor morbidade, e retorno precoce às atividades. ${ }^{11}$

O presente estudo avaliou os resultados clínicos e funcionais da discectomia endoscópica transforaminal em pacientes portadores de hérnia de disco lombar.

\section{Materiais e Métodos}

Entre agosto de 2015 e janeiro de 2017, 101 pacientes foram submetidos a discectomia endoscópica transforaminal lombar devido a hérnia de disco lombar refratária a tratamento clínico. Este estudo foi submetido e aprovado pelo Comitê de Ética da Faculdade de Medicina do ABC (CAAE 85551418.0.000.0082). Todos os pacientes assinaram o termo de consentimento livre e esclarecido.
Foram incluídos pacientes com hérnia de disco determinada por meio das imagens de ressonância magnética associadas a teste positivo de tensão da raiz nervosa no exame físico, e dor ciática persistente por mais de seis semanas de tratamento conservador adequado (fisioterapia, analgesia, repouso).

Estenose lombar grave, instabilidade (escorregamento maior do que $3 \mathrm{~mm}$ em relação à vértebra adjacente durante radiografias de flexão e extensão), tumor, trauma, e infecção foram os critérios de exclusão.

Os pacientes foram avaliados quanto aos dados clínicos para dor por meio da Escala Visual Analógica (EVA) e quanto aos achados funcionais pelo Oswestry Disability Index (ODI). Esses dados foram obtidos no período pré-operatório, no pós-operatório imediato, com um mês, três meses, seis meses, e um anos após a cirurgia. Durante o seguimento pós-operatório, os pacientes que evoluíram com persistência ou novos sintomas de dor radicular foram submetidos a nova ressonância magnética.

Foi adotado o nível de significância de $5 \%(0,050)$ para a aplicação dos testes estatísticos, ou seja, quando o valor da significância calculada $(p)$ foi menor do que $5 \%$, observou-se uma diferença ou relação estatisticamente significativa; em contrapartida, quando o valor $p$ foi maior do que $5 \%$, identificou-se uma diferença ou relação dita estatisticamente não significativa. Foi utilizada a planilha eletrônica Excel do pacote Office 2013 (Microsoft Corp., Redmond, WA, EUA) para a organização dos dados, e o programa Statistical Package for the Social Sciences (SPSS, IBM Corp., Armonk, NY, EUA), versão 24.0, para a obtenção dos resultados. O Teste de MannWhitney foi aplicado com o intuito de verificar possíveis diferenças entre as primeiras linhas (casos operados) e as últimas 30 linhas para as variáveis de interesse. 0 teste dos Postos Sinalizados de Wilcoxon foi usado para identificar quais momentos de observação diferiam dos demais.

Em relação à técnica cirúrgica, o paciente é posicionado em decúbito ventral numa mesa radiotransparente, com o quadril e joelhos fletidos, sob sedação consciente. Após assepsia adequada, a marcação da pele é realizada com auxílio da visualização da fluoroscopia. Em seguida, aplica-se uma anestesia local com xilocaína sem vasoconstritor a $1 \%$ no local estabelecido da punção. É introduzida uma agulha de 18 gauge até a porção lateral da faceta e, depois, na face posterior do 

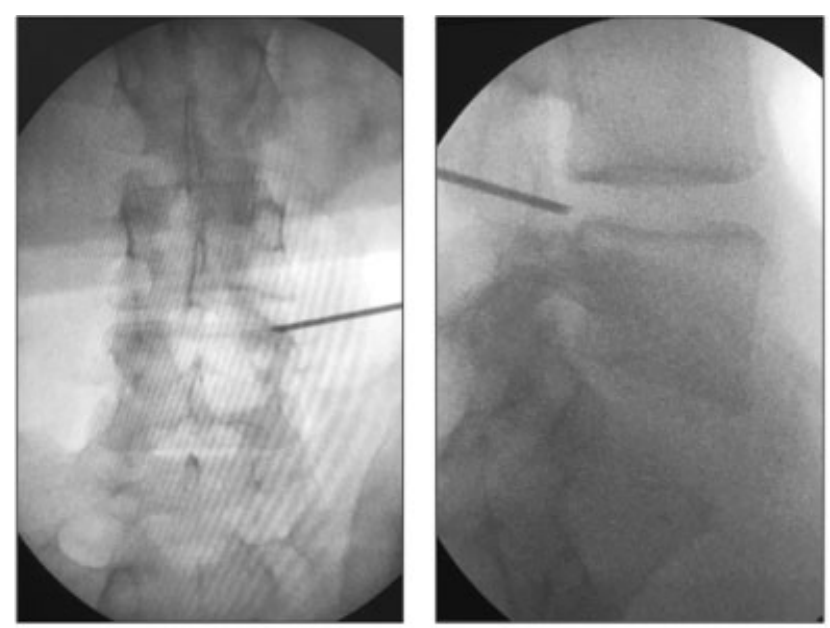

Fig. 1 Inserção da agulha na projeção anteroposterior e em perfil.

ânulo fibroso, locais onde é aplicado mais anestésico. Neste momento, observa-se por meio do intensificador de imagem a ponta da agulha localizada na linha pedicular medial na projeção anteroposterior e na borda posterior vertebral, na projeção em perfil (- Figura 1).

Realiza-se uma discografia com uma solução de contraste não iodado e azul de metileno. Em seguida, fio guia, dilatador e cânula de trabalho são introduzidos, para, então, um endoscópio com 30 graus de angulação e irrigação de fluxo contínuo ser instalado. Em caso de sangramento, hemostasia é feita com um coagulador bipolar. O fragmento de disco herniado é identificado com coloração azul, e delicadamente retirado com pinças específicas (- Figuras 2 e 3 ). Geralmente, é possível observar a liberação da raiz nervosa com oscilação

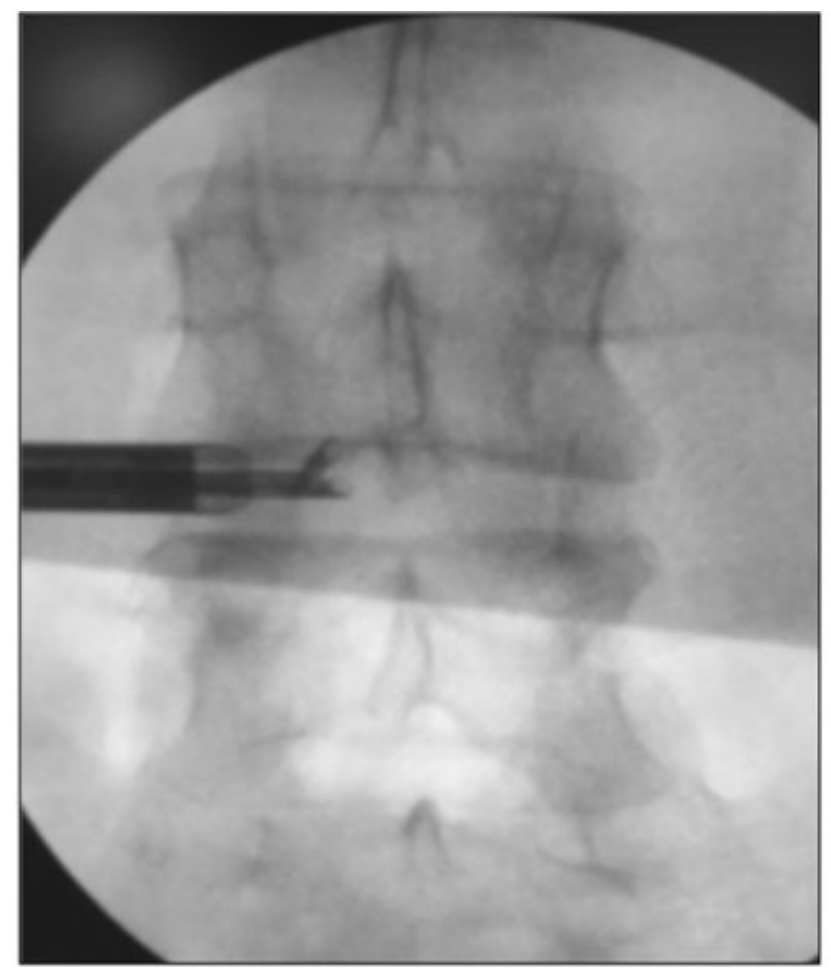

Fig. 2 Imagem da pinça de disco no espaço discal.

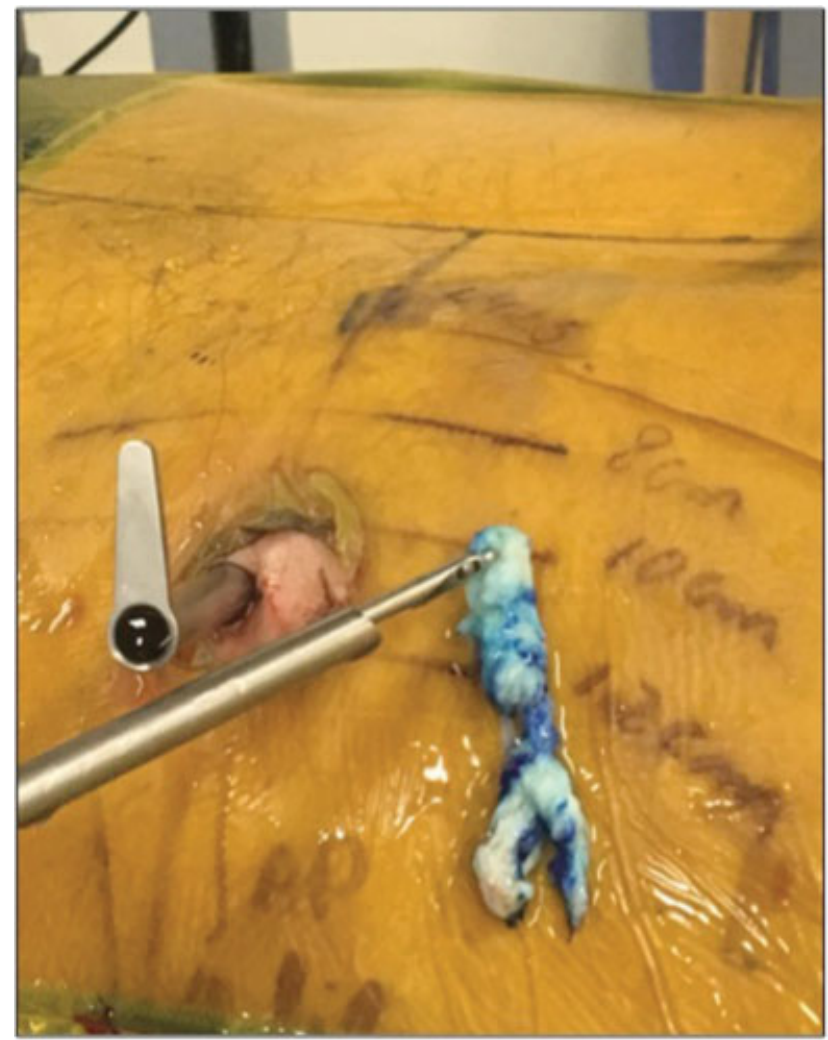

Fig. 3 Remoção do fragmento discal extruso.

da pressão da irrigação. Os pacientes são liberados para deambular assim que há total recuperação da sedação.

\section{Resultados}

De 107 pacientes operados, um total de 101 (94,4\%) pacientes foram incluídos no estudo, sendo 6 pacientes excluídos por perda de seguimento pós-operatório. A média de idade dos participantes foi de 48.1 anos (variando de 20 a 78 anos). A amostra foi composta de 55 homens e 46 mulheres. A - Tabela 1 mostra os dados demográficos dos pacientes. Os níveis discais mais acometidos foram L4-L5, seguidos de L5-S1. Um total de 29 pacientes foram abordados em 2 níveis discais.

A duração do procedimento cirúrgico variou 36 a 126 minutos (média de 54 minutos). Ao todo, 82 pacientes

Tabela 1 Características demográficas e clínicas

\begin{tabular}{|l|l|}
\hline Variáveis & Valores \\
\hline Número de pacientes & 101 \\
\hline Idade (média) & 48,1 \\
\hline Masculino/Feminino & $55 / 46$ \\
\hline $\begin{array}{l}\text { Média do índice de massa } \\
\text { corporal (kg/m²) }\end{array}$ & 28,2 \\
\hline Nível discal cirúrgico & \\
\hline L3-L4 & 2 \\
\hline L4-L5 & 19 \\
\hline L5-S1 & 11 \\
\hline
\end{tabular}


Tabela 2 Questionário de Escala Visual Analógica (EVA) e Oswestry Disability Index (ODI)

\begin{tabular}{|l|l|l|l|l|}
\hline Período & \multicolumn{4}{l|}{ Avaliação } \\
\hline & EVA & $p^{* * *}$ & ODI & $p^{* * *}$ \\
\hline Pré-operatório & 16,37 & & 15,51 & \\
\hline $\mathbf{1}$ semana & 6,15 & $<0,001$ & 7,62 & $<0,001$ \\
\hline $\mathbf{1}$ mês & 5,42 & $<0,001$ & 5,77 & $<0,001$ \\
\hline $\mathbf{3}$ meses & 4,00 & $<0,001$ & 3,82 & $<0,001$ \\
\hline $\mathbf{6}$ meses & 3,81 & $<0,001$ & 3,74 & $<0,001$ \\
\hline $\mathbf{1 2}$ meses & 3,76 & $<0,001$ & 3,46 & $<0,001$ \\
\hline
\end{tabular}

Nota: ${ }^{* * *} p<0.05=$ diferença estatisticamente significativa (comparação com o período pré-operatório).

tiveram alta hospitalar no mesmo dia; entretanto, 19 pacientes foram operados no período da noite, e, portanto, receberam alta hospitalar na manhã seguinte. Nenhum paciente teve mais de um dia de internação hospitalar, e $91 \%$ dos procedimentos foram realizados sob anestesia local e sedação. Um total de $5 \%$ dos pacientes relataram medo de procedimentos com manutenção da consciência, optando por anestesia geral. Nestes pacientes, a cirurgia não foi realizada sob monitoração neurofisiológica; entretanto, optamos por incluí-los no estudo, haja vista que os casos envolviam hérnias discais em níveis com espaços foraminais amplos (L3-L4 e L4-L5), com menor risco de lesão da raiz nervosa emergente.

Os achados clínicos e funcionais do pré-operatório e do seguimento pós-operatório podem ser observados na - Tabela 2. Após 1 mês de seguimento pós-operatório, a média das pontuações nos questionários (EVA e ODI) diminuiu significativamente $(p<0.001)$. Essa diminuição permaneceu na avaliação 12 meses após a cirurgia em relação à pontuação inicial $(p<0.001)$, mas sem diferença estatística na comparação com as pontuações com um mês de seguimento.

A - Tabela 3 retrata a comparação dos achados clínicos e funcionais doze meses após o procedimento dos trinta

Tabela 3 Comparação dos achados clínicos e funcionais 12 meses após o procedimento dos primeiros 30 pacientes em relação aos últimos 30 pacientes

\begin{tabular}{|l|l|l|l|l|}
\hline Variável & Categoria & $\mathbf{n}$ & Média & $p^{* * *}$ \\
\hline $\begin{array}{l}\text { Escala Visual } \\
\text { Analógica }\end{array}$ & $\begin{array}{l}\text { Primeiros } \\
30 \text { pacientes }\end{array}$ & 30 & 6,00 & 0,444 \\
\hline & $\begin{array}{l}\text { Últimos } \\
30 \text { pacientes }\end{array}$ & 30 & 2,90 & \\
\hline & Total & 60 & & 0,830 \\
\hline $\begin{array}{l}\text { Oswestry } \\
\text { Index }\end{array}$ & $\begin{array}{l}\text { Primeiros } \\
30 \text { pacientes }\end{array}$ & 30 & 5,4 & \\
\hline & $\begin{array}{l}\text { Últimos } \\
30 \text { pacientes }\end{array}$ & 30 & 2,83 & \\
\hline & Total & 60 & & \\
\hline
\end{tabular}

Nota: ${ }^{* * *} p<0.05=$ diferença estatisticamente significantiva.
Tabela 4 Comparação dos achados clínicos e funcionais 12 meses após os procedimentos dos níveis discais que não incluíam L5-S1 em relação aos que incluíam o nível discal L5-S1

\begin{tabular}{|l|l|l|l|l|}
\hline Variável & Categoria & $\mathbf{n}$ & Média & $p^{* * *}$ \\
\hline $\begin{array}{l}\text { Escala Visual } \\
\text { Analógica }\end{array}$ & Sem L5-S1 & 40 & 3,93 & 0,997 \\
\hline & Com L5-S1 & 61 & 3,66 & \\
\hline & Total & 101 & 3,76 & \\
\hline $\begin{array}{l}\text { Oswestry } \\
\text { Disability Index }\end{array}$ & Sem L5-S1 & 40 & 3,63 & 0,950 \\
\hline & Com L5-S1 & 61 & 3,34 & \\
\hline & Total & 101 & 3,46 & \\
\hline
\end{tabular}

Nota: ${ }^{* * *} p<0.05=$ diferença estatisticamente significativa.

pacientes inicialmente operados em relação aos últimos trinta pacientes.

A análise dos achados clínicos e funcionais 12 meses após os procedimentos que envolviam os níveis discais L3-L4 e L4-L5 em relação aos procedimentos envolvendo o nível discal L5-S1 foi descrita na - Tabela 4.

\section{Discussão}

Durante muitos anos, a cirurgia aberta convencional foi considerada o "padrão-ouro" para o tratamento da hérnia de disco intervertebral. Com o advento da microdiscectomia, houve uma redução significativa da morbidade da cirurgia aberta, do tempo de internação hospitalar e da perda sanguínea. ${ }^{12}$ Mesmo assim, a microdiscectomia apresenta algumas desvantagens em comum com a cirurgia aberta, como retração da musculatura paravertebral, dor pós-operatória, ressecção óssea, além dos risco de complicações em longo prazo (recorrência, fibrose epidural, instabilidade vertebral), as quais são desafios para um cirurgião experiente. ${ }^{13}$ Os resultados da microdiscectomia lombar nos prolapsos discais recorrentes não são bons quando comparados com os dos casos primários. ${ }^{14}$

Diante dessas limitações, a discectomia endoscópica percutânea por via transforaminal é uma alternativa usada para tratar herniações de disco lombares. Os avanços nos instrumentos permitiram uma visualização endoscópica direta para uma remoção segura do material discal. ${ }^{15}$

Em 2002, Yeung e Tsou ${ }^{16}$ descreveram resultados da excisão discal em 307 pacientes, e observaram 89.7\% de resultados satisfatórios após 1 ano de seguimento. As complicações foram infecção profunda em dois pacientes, tromboflebite em dois pacientes, disestesia em seis pacientes, e lesão dural em um paciente.

Em 2008, Ruetten et al $^{17}$ realizaram um estudo prospectivo randomizado controlado comparando microdiscectomia e endoscopia em 178 pacientes. Observaram resultados similares, que foram satisfatórios em $96 \%$ dos casos. Entretanto, o procedimento endoscópico apresentou vantagens significativas com relação à microdiscectomia em termos de tempo cirúrgico, custo, reabilitação, dor lombar, cicatriz, revisão precoce e complicações. 
Chae et al $^{18}$ relataram $94,77 \%$ de resultados satisfatórios com discectomia endoscópica percutânea em 153 pacientes com hérnia discal lombar extrusa. Obtiveram 96\% de resultados bons e excelentes, e uma taxa de complicações extremamente baixa, sem nenhum caso de infecção de ferida operatória.

Em 2010, Jhala e Mistry ${ }^{9}$ relataram resultados de discectomia endoscópica em 100 pacientes, com 91\% de resultados bons e excelentes. Os autores observaram sete lesões durais, uma lesão nervosa e quatro pacientes com recorrência, quatro discites, e cinco remoções da faceta. Quatro pacientes tiveram que reoperar.

Em 2012, Kaushal e Sen ${ }^{19}$ relataram resultados satisfatórios em $90 \%$ dos pacientes após discectomia endoscópica em 300 casos. Entre as complicações, observaram cinco lesões da dura-máter, cinco discites, e duas lesões de raízes nervosas. Em 2014, Kulkarni et al ${ }^{15}$ observaram resultados da discectomia endoscópica em 188 pacientes, e verificaram 5\% de lesão dural, $2.1 \%$ de hérnia de disco residual, $1.5 \%$ de recorrência, $0.5 \%$ de nível errado, e $0.5 \%$ de infecção.

Uma metanálise $\mathrm{e}^{20}$ comparou discectomia endoscópica e microdiscectomia aberta, e nela observou-se uma maior taxa de satisfação entre os pacientes submetidos ao procedimento endoscópico. Além disso, a abordagem percutânea estava associada a menor perda sanguínea e curto período de hospitalização, mas não houve diferença estatística em relação ao tempo cirúrgico, recorrência ou taxas de complicações. $^{20}$

Em uma revisão retrospectiva de 10,228 casos em um único centro, Choi et $\mathrm{al}^{21}$ estudaram a causa da falha da discectomia endoscópica transforaminal no tratamento da hérnia de disco lombar. Observaram uma taxa de insucesso de $4.3 \%$, sendo a remoção incompleta do material discal o principal motivo (2.8\%), seguida de recorrência da hérnia discal em $0.8 \%$, e persistência da dor após completa remoção do fragmento $(0.4 \%)^{21}$

A maior desvantagem da discectomia endoscópica é a longa curva de aprendizado. Em 2013, Wang et al ${ }^{22}$ recomendaram a prática de infiltração epidural transforaminal antes de realizar o procedimento endoscópico. ${ }^{22}$ Em 2016, Wu et al $^{23}$ compararam os primeiros 60 casos de discectomia endoscópica transforaminal no nível L4-L5 (grupo I) com os primeiros 60 casos no nível L5-S1 (grupo II). Perceberam que a curva de aprendizado no nível L5-S1 foi mais difícil, haja vista as particularidades anatômicas, como a altura da crista ilíaca, articulação facetaria larga, processo transverso de L5 largo, espaço discal estreito, e menor dimensão do espaço foraminal. Além disso, observaram uma diferença estatística quando comparado o tempo cirúrgico dos primeiros 20 casos com os últimos 20 casos em ambos os grupos. Hsu et $\mathrm{al}^{24}$ demonstraram que a curva de aprendizado na abordagem transforaminal era mais fácil do que o acesso interlaminar. Apesar de os cirurgiões de coluna estarem mais familiarizados com a anatomia posterior, o procedimento transforaminal requer em média menos tempo cirúrgico do que o procedimento interlaminar.

No presente estudo, não foram observadas lesão dural incidental, lesão neurológica permanente, ou infecção discal ou do sítio cirúrgico. A baixa taxa de infecção pode ser explicada por danos mínimos ao tecido normal, menos sangramento, tempo operatório curto, irrigação salina contínua, e técnica cirúrgica. Houve três casos de recidiva da hérnia discal no mesmo nível após um período de melhora expressiva dos sintomas. Desses casos, apenas um paciente necessitou de uma nova discectomia endoscópica. Conversão para microdiscectomia aberta não foi necessária em nenhum caso, nem na recorrência da hérnia de disco. Dor ciática tolerável foi observada em cinco pacientes, com boa melhora após uma média de quatro semanas de tratamento conservador. Tais complicações apresentaram taxas semelhantes às apresentadas na literatura. Observamos que as complicações ocorreram nos primeiros trinta casos iniciais, mas sem diferença estatística quando comparados com os últimos trinta casos. E tampouco identificamos diferença estatística nos achados em relação ao nível discal operado.

Os resultados clínicos e funcionais foram considerados os mais importantes no seguimento após a discectomia, e, portanto, não foi estudada a diminuição da hérnia de disco no pós-operatório por meio de exames de imagem, pois a ressonância magnética foi realizada apenas para os casos de persistência ou de novos sintomas de ciatalgia. Estudos randomizados controlados com maior número de pacientes e maiores intervalos de acompanhamento são necessários para avaliar objetivamente o impacto da discectomia endoscópica.

\section{Conclusão}

A discectomia endoscópica transforaminal lombar mostrou ser uma alternativa segura, eficaz e minimamente invasiva para o tratamento de hérnia de disco lombar. Embora necessite de uma longa curva de aprendizado, a técnica apresenta vantagens, como mínimo dano das partes moles, curto tempo cirúrgico e de internação hospitalar, baixa taxa de complicações, e retorno precoce ao trabalho.

Conflito de Interesses

Os autores declaram não haver conflito de interesses.

\section{Referências}

1 Andersson GB. Epidemiological features of chronic low-back pain. Lancet 1999;354(9178):581-585

2 Papageorgiou AC, Croft PR, Ferry S, Jayson MI, Silman AJ. Estimating the prevalence of low back pain in the general population. Evidence from the South Manchester Back Pain Survey. Spine 1995;20(17):1889-1894

3 Konstantinou K, Dunn KM. Sciatica: review of epidemiological studies and prevalence estimates. Spine 2008;33(22):2464-2472

4 Spangfort EV. The lumbar disc herniation. A computer-aided analysis of 2,504 operations. Acta Orthop Scand Suppl 1972; 142:1-95

5 Mixter WJ, Barr J. Rupture of the intervertebral disc with involvement of the spinal canal. N Engl J Med 1934;211:208-215

6 Hijikata S. Percutaneous nucleotomy. A new concept technique and 12 years' experience. Clin Orthop Relat Res 1989;(238):9-23

7 Kambin P, Schaffer JL. Percutaneous lumbar discectomy. Review of 100 patients and current practice. Clin Orthop Relat Res 1989; (238):24-34 
8 Foley KT, Smith MM. Microendoscopic Discectomy. Tech Neurosurg 1997;3:301-307

9 Jhala A, Mistry M. Endoscopic lumbar discectomy: Experience of first 100 cases. Indian J Orthop 2010;44(02):184-190

10 Yeung AT. Minimally Invasive Disc Surgery with the Yeung Endoscopic Spine System (YESS). Surg Technol Int 1999;8:267-277

11 Maroon JC. Current concepts in minimally invasive discectomy. Neurosurgery 2002;51(5, Suppl)S137-S145

12 Caspar W. A new surgical procedure for lumbar disc herniation causing less tissue damage through microsurgical approach. In: Wullenweber R, Brock M, Hamer J, Klinger M, Spoerri O, editors. Advances in Neurosurgery. Berlin: Springer-Verlag; 1977:74-77

13 Kraemer R, Wild A, Haak H, Herdmann J, Krauspe R, Kraemer J. Classification and management of early complications in open lumbar microdiscectomy. Eur Spine J 2003;12(03):239-246

14 Acharya KN, Nathan TS, Kumar JR, Menon KV. Primary and revision lumbar discectomy: a three-year review from one center. Indian J Orthop 2008;42(02):178-181

15 Kulkarni AG, Bassi A, Dhruv A. Microendoscopic lumbar discectomy: Technique and results of 188 cases. Indian J Orthop 2014; 48(01):81-87

16 Yeung AT, Tsou PM. Posterolateral endoscopic excision for lumbar disc herniation: Surgical technique, outcome, and complications in 307 consecutive cases. Spine 2002;27(07):722-731

17 Ruetten S, Komp M, Merk H, Godolias G. Full-endoscopic interlaminar and transforaminal lumbar discectomy versus conventional microsurgical technique: a prospective, randomized, controlled study. Spine 2008;33(09):931-939

18 Chae KH, Ju CI, Lee SM, Kim BW, Kim SY, Kim HS. Strategies for Noncontained Lumbar Disc Herniation by an Endoscopic Approach : Transforaminal Suprapedicular Approach, Semi-Rigid Flexible Curved Probe, and 3-Dimensional Reconstruction CT with Discogram. J Korean Neurosurg Soc 2009;46(04):312-316

19 Kaushal M, Sen R. Posterior endoscopic discectomy: Results in 300 patients. Indian J Orthop 2012;46(01):81-85

20 Cong L, Zhu Y, Tu G. A meta-analysis of endoscopic discectomy versus open discectomy for symptomatic lumbar disk herniation. Eur Spine J 2016;25(01):134-143

21 Choi KC, Lee JH, Kim JS, et al. Unsuccessful percutaneous endoscopic lumbar discectomy: a single-center experience of 10,228 cases. Neurosurgery 2015;76(04):372-380, discussion 380-381, quiz 381

22 Wang H, Huang B, Li C, et al. Learning curve for percutaneous endoscopic lumbar discectomy depending on the surgeon's training level of minimally invasive spine surgery. Clin Neurol Neurosurg 2013;115(10):1987-1991

$23 \mathrm{Wu} \mathrm{XB}$, Fan GX, Gu X, et al. Learning curves of percutaneous endoscopic lumbar discectomy in transforaminal approach at the L4/5 and L5/S1 levels: a comparative study. J Zhejiang Univ Sci B 2016;17(07):553-560

24 Hsu HT, Chang SJ, Yang SS, Chai CL. Learning curve of fullendoscopic lumbar discectomy. Eur Spine J 2013;22(04):727-733 